\title{
Lo stalking, un reato senza genere
}

\author{
Annamaria Iaccarino
}

\section{Riassunto}

Colui che si apposta, insegue, pedina e controlla la vittima per intromettersi, più o meno violentemente, nella sua vita privata viene definito stalker o "cacciatore dell'agguato" perché realizza una serie ripetuta di azioni o di tentativi di contatti o di effettive comunicazioni, che sono di fatto indesiderati, provocando disagio, angoscia, fastidio, preoccupazione e paura. Il delitto di stalking è un reato compiuto senza alcuna differenza di genere.

Alla "Donna Mostro" di Lombroso e alla "Follia delle zitelle" di Hart si accosta il fenomeno dello stalking femminile le cui modalità operative sono sicuramente più subdole e sottili rispetto a quelle maschili, richiamando alla mente quell'agire retrospettivo analizzato dai teorici classici.

\section{Résumé}

Celui qui surveille, poursuit, piste et contrôle la victime dans le but de s'entremettre, plus ou moins violemment, dans sa vie privée est qualifié de harceleur ou de «chasseur aux aguets ", puisqu'il commet une série d'actions de façon répétée ou de tentatives de contact ou de véritables communications, non désirées, qui provoquent gêne, angoisse, désagrément, préoccupation et peur chez la victime. Le crime de harcèlement est commis par tout le monde sans aucune distinction fondée sur le genre.

À la «femme monstre » de Lombroso et à la «folie des vieilles filles » de Hart s'ajoute le phénomène du harcèlement commis par des femmes dont le mode opératoire est, sans aucun doute, plus sournois et plus subtil que celui des hommes. Il évoque l'agissement rétrospectif analysé par les classiques.

\section{Abstract}

An individual who watches, follows, tracks and controls the victim with the aim of intervening, in a more or less violent way, in their private life is called a stalker or a "prowler always on the lookout", because they commit repeated actions or attempted contacts or real communications, not desired by the victim, which may cause discomfort, anxiety, nuisance, concern and fear to the victim. Stalking is a term that may apply to any gender.

To Lombroso's "monster woman" and Hart's "old maids' madness" it is necessary to add the phenomenon of stalking committed by women whose modus operandi, without doubt, is more sly and subtle than the one of men. It refers to the retrospective act analysed by classic theories.

Key words: stalking; gender ; modus operandi ; victim ; fear.

\footnotetext{
- Criminologo, Avvocato specializzato in Diritto e Procedura penale, Dottore di ricerca in Criminologia, Devianza e Mutamento sociale, Cultore della materia e Collaboratore di Cattedra in Criminologia presso l'Università degli Studi Suor Orsola Benincasa di Napoli.
} 


\section{Lo stalking al femminile.}

L'introduzione legislativa del delitto di stalking costituisce una grande vittoria, non solo giuridica, ma anche criminologica e sociale.Prima della legge n. 38 del 23 aprile 2009, con cui si convertiva il D.l. n.11 del 23 febbraio 2009, il nostro codice penale prevedeva solo il reato di minaccia ${ }^{1}$, che non poteva estendersi ed applicarsi a tutte le fattispecie penalmente rilevanti invece contemperate dall'art. 612 bis c. $p^{2}$.

Con questa disposizione normativa, infatti, il Legislatore è voluto intervenire con delle "Misure urgenti in materia di sicurezza pubblica e di contrasto alla violenza sessuale", nonché in

${ }^{1}$ Art. $612 \mathrm{cp}$ "Chiunque minaccia ad altri un ingiusto danno è punito, a querela della persona offesa, con la multa fino a 1.032 euro.

Se la minaccia è grave, o è fatta in uno dei modi indicati nell'articolo 339, la pena è della reclusione fino a un anno e si procede d'ufficio".

2 Art 612 bis cp "Salvo che il fatto costituisca più grave reato, è punito con la reclusione da sei mesi a cinque anni chiunque, con condotte reiterate, minaccia o molesta taluno in modo da cagionare un perdurante e grave stato di ansia o di paura ovvero da ingenerare un fondato timore per l'incolumità propria o di un prossimo congiunto o di persona al medesimo legata da relazione affettiva ovvero da costringere lo stesso ad alterare le proprie abitudini di vita.

La pena è aumentata se il fatto è commesso dal coniuge legalmente separato o divorziato o da persona che sia stata legata da relazione affettiva alla persona offesa ovvero se il fatto è commesso attraverso strumenti informatici o telematici.

La pena è aumentata fino alla metà se il fatto è commesso a danno di un minore, di una donna in stato di gravidanza o di una persona con disabilità di cui all'art. 3 della legge 5.2.1992 n. 104, ovvero con armi o da persona travisata.

Il delitto è punito a querela della persona offesa. Il termine per la proposizione della querela è di sei mesi. Si procede tuttavia di ufficio se il fatto è commesso nei confronti di un minore o di una persona con disabilità di cui all'art. 3 legge 5.2.1992, n.104, nonché quando il fatto è connesso con altro delitto per il quale si deve procedere di ufficio. La remissione della querela può essere soltanto processuale. La querela è comunque irrevocabile se il fatto è stato commesso mediante minacce reiterate nei modi di cui all'articolo 612, secondo comma". tema di atti persecutori lesivi della sfera giuridica individuale.

Bisogna tener presente che ogni anno circa 70.000 donne sono vittime di stupri, tentati stupri e di atti persecutori in generale ${ }^{3}$, che necessitavano di una previsione normativa ad hoc, che incriminasse le condotte reiterate di molestia e di minaccia responsabili di rilevanti disagi psichici per la persona offesa.

Prima della legge n. 38 del 2009, e al di fuori del delitto di minaccia, le condotte di petulanza e di disturbo trovavano come unica tutela l'applicazione della contravvenzione ex art. 660 $\mathrm{cp}^{4}$, che tendeva a punire quei comportamenti idonei a turbare in modo apprezzabile le normali condizioni di vita della persona molestata ${ }^{5}$, in questo caso però il bene giuridico tutelato non era quello individuale, fisico o psichico, della vittima, ed in più, trattandosi di una contravvenzione ${ }^{6}$, vi era un inferiore tempo di prescrizione 7 .

Prodromica della riforma è stata, tra le altre, l'indagine Istat degli anni $2006^{8}$, interamente dedicata al fenomeno delle violenza fisica e sessuale sulle donne; ricerca questa che ha denunciato il maggior numero di reati consumati e tentati degli ultimi dieci anni.

3 Disponibile alla pagina: www.istat/dati/catalogo.pdf.

4 Art 660 cp "Chiunque, in un luogo pubblico o aperto al pubblico, ovvero col mezzo del telefono, per petulanza o per altro biasimevole motivo, reca a taluno molestia o disturbo è punito con l'arresto fino a sei mesi o con l'ammenda fino a euro 516".

${ }^{5}$ Corte di Cassazione, IV ${ }^{\circ}$ sez. pen. n. 2967 del 1978.

${ }^{6}$ In particolare una contravvenzione contro l'ordine pubblico.

7 Tra l'altro il locus commissi delicti configurato per volontà della norma si esauriva esclusivamente se il reato avveniva in un luogo pubblico o aperto al pubblico.

${ }^{8}$ Disponibile alla pagina: www.istat.it. 
Sulle molestie e violenze sessuali erano già state fatte dall'Istat delle considerazioni nel 1997 e poi nel 2002 nell'ambito dell'indagine "Multiscopo sulla sicurezza dei cittadini", considerazioni che hanno confermato quanto riportato successivamente dal campione comprendente 25 mila donne, tra i 16 e i 70 anni, intervistate su tutto il territorio nazionale dal gennaio all'ottobre 2006 con tecnica telefonica'.

Questa indagine sulla sicurezza femminile, ricercando i diversi tipi di violenza (fisica, sessuale e psicologica ${ }^{10}$ ) sia dentro la famiglia che fuori dalla stessa, non ha fatto emergere particolari forme di violenza persecutoria. Infatti, non si sono registrate le molestie verbali, il pedinamento, gli atti di esibizionismo e le telefonate oscene. Allo stesso tempo, però, dalle forme di violenza psicologica si rilevavano numerosi casi di denigrazione, controllo dei comportamenti, strategie di isolamento, intimidazioni ed, infine, forti limitazioni economiche subite da parte del partner ${ }^{11}$.

Come già noto tra gli addetti ai lavori, relativamente ad ipotesi di violenza in famiglia, dalla tavola n. 10 del rapporto di ricerca emerge che solo il $7,3 \%$ ha deciso di denunciare $i$ comportamenti delittuosi, mentre il $92,4 \%$, per le

\footnotetext{
9 L'indagine è frutto di una convenzione tra l'Istat, che l'ha condotta, e il Ministero per i Diritti e le Pari Opportunità, che l'ha finanziata con i fondi del Programma Operativo Nazionale "Sicurezza" e "Azioni di sistema" del Fondo Sociale Europeo. Ivi.

10 La violenza fisica è stata analizzata graduandola dalle forme più lievi a quelle più gravi delle lesioni o tentato omicidio. Ivi.

La violenza sessuale è stata ricercata sia in base alla presenza dei classici atti sessuali intesi in senso stretto, sia con quelli di maggior disvalore, quali lo stupro, il tentato stupro, la molestia fisica sessuale, i rapporti sessuali con terzi, i rapporti sessuali non desiderati subiti per paura delle conseguenze, le attività sessuali degradanti e umilianti, Ivi.
}

stesse violenze fisiche e sessuali subite, ha preferito alimentare il già elevato numero oscuro in tema ${ }^{12}$.

Il tasso di denuncia è alquanto basso, pari cioè al $12,4 \%$, nonostante il parlarne in famiglia. Questa criticità viene superata, in un certo senso, se si pensa che l'indice della denuncia è più elevato quando le donne si rivolgono ad operatori del pronto soccorso (62,3\%), ad avvocati, magistrati, polizia, carabinieri $(47,6 \%)$ ad un medico o infermiere $(35,9 \%)^{13}$.

La gravità della violenza non incide su un maggior ricorso alla denuncia. Solo il 5,3\% degli stupri o tentati stupri è stato denunciato. Alla quota delle denunce va aggiunto un $2,6 \%$ di donne che hanno subito ripetutamente violenza e che pur non avendo denunciato hanno telefonato al 112 e 113 per cercare aiuto.

L'ampia casistica di dati ${ }^{14}$, che ha sollevato le problematiche sul "femminicidio", ispirando i lavori preparatori della legge, conclusa con la programmazione del delitto di stalking, a 6 anni dalla sua nascita, non mostra una particolare differenza di genere in relazione agli autori del reato e ai suoi destinatari.

Il delitto di stalking non appartiene ad un genere specifico, perché realizzato indifferentemente da uomini e da donne.

Non è facile tracciare percentuali affidabili del fenomeno dello stalking femminile per la diversità dei campioni di studio fatta anche a livello internazionale.

\footnotetext{
11 Disponibile alla pagina:

www.istat.it/giustizia/sicurezza.

${ }^{12} \mathrm{Ivi}$.

${ }^{13} \mathrm{Ivi}$.

${ }^{14}$ Istat, denunzie, procedimenti penali conclusi e non sull'analisi di reati di omicidio, consumato e tentato, violenza sessuale e lesioni in cui il delitto è stato preceduto da molestie assillanti.
} 
In Germania, indagini condotte attraverso un questionario inviato per posta a un campione rappresentativo di cittadini hanno dimostrato che il $14,5 \%$ ha come autore una donna ${ }^{15}$.

Percentuali più elevate emergono da studi condotti nelle "Criminal Court Population" o in ambito giudiziario americano. Secondo un'indagine condotta presso la New York County Criminal and Supreme Court, il 33\% degli stalkers risultava essere di genere femminile ${ }^{16}$.

Ancora, secondo uno studio retrospettivo fatto dal dipartimento di Polizia di Los Angeles Police $^{17}$ sembra emergere che nel $32 \%$ dei casi gli autori delle condotte di stalking fosserodonne, molte delle quali affette da delirio erotomanico.

Ancora nell'ambito degli studi di vittimizzazione è interessante ricordare la ricerca di Sheridan e Davies $^{18}$, svolta su 95 vittime di stalking afferite al Suzy Lamplugh Trust, in un centro di promozione sociale e sanitaria con sede a Londra. Proprio da questa ricerca è emerso che

${ }^{15}$ Dressing H., Kuehner C., Gass P., "Prevalence of stalking in Germany", Psychiatrische Praxis, 32 (2), 2005, pp. 73-78; Dressing H., Kuehner C., Gass P., "Lifetime prevalence and impact of stalking in a European population: epidemiological data from a middle-sized German city", British Journal of Psychiatry, 187, 2005, pp. 168-172; Dressing H., Kuehner C., Gass P., "The epidemiology and characteristics of stalking”, Current opinion in psychiatry, 19 (4), 2006, pp. 395-399.

${ }^{16}$ In Harmon R., Rosner R, Owens H., "Sex and violence in a forensic population of obsessional harassers", Psychology, Public Policy and Law, vol. 4, no. 1.2, 1998. pp. 236-49.

${ }_{17}$ Los Angeles Police Department Zona, Sharma \& Lane, 1993, in Alfarano E., La Tegola D., Carabellese F., Catanesi R., Stalking al femminile: una review di letteratura in Rassegna italiana di Criminologia, anno IV, n.1, 2012.

18 Sheridan L., Davies G., Bonn J., "The Course and Nature of Stalking: A victim Perspective", in The Howard Journal, 2001, pp. 215 -234. nel $7 \%$ dei casi si poteva parlare di stalking femminile.

In tempi più recenti Spitzberg ${ }^{19}$ ha indagato su campioni clinico-forensi. Ha analizzato studi di popolazione generale ed anche campioni di studenti di college di circa trenta stati USA, evidenziando che la percentuale di stalking femminile varia fra il $2 \%$ e il $13 \%$.

\section{I bisogni e $\mathrm{i}$ desideri nelle diverse tipologie di stalker.}

"To stalk", che letteralmente significa proprio "appostarsi”, rende alla perfezione l'idea dello stalker che si apposta, insegue, pedina e controlla la sua vittima per intromettersi, più o meno violentemente, nella sua vita privata. Non è un caso che la parola stalking possa essere tradotta come "cacciatore dell'agguato" per colui o colei che avanzano furtivamente nella vita altrui.

La sindrome del molestatore assillante si realizza quando il soggetto attore pone in essere una serie ripetuta di azioni o di tentativi di contatti o di effettive comunicazioni, che sono di fatto indesiderati per la vittima, provocando disagio, angoscia, fastidio, preoccupazione e paura.

Secondo Mullen ${ }^{20}$ per le cinque tipologie di stalkers ci sono diversi bisogni e desideri che fanno da motore motivazionale.

"Il risentito", ad esempio, agisce perché spinto dal desiderio di vendicarsi di un torto che ritiene aver subito, essendo alimentato dalla ricerca di vendetta. Questa categoria risulta piuttosto pericolosa perché finalizzata alla lesione non solo

\footnotetext{
19 Spitzberg B., "The state of the art of stalking: taking stock of the emerging literature", in Aggression and Violent Behavior, 2007, pp. 64-86.

${ }^{20}$ Mullen P.E., "The victim of stalking", in Boon J., Sheridan L. (Eds.), Stalking and Psychosexual Obsession:
} 
dell'immagine dell'individuo, ma proprio della persona stessa.

Il movente principale risulta essere il risentimento in grado di giustificare $i$ comportamenti persecutori, meccanismo che richiama alla mente le tecniche di neutralizzazione spiegateci da Matza ${ }^{21}$, secondo cui le strategie cognitive, di tipo passivo, sono messe in atto dagli attori criminali o, più in generale, da devianti, allo scopo di fronteggiare le conseguenze psicologiche derivanti dall'aver posto in essere una condotta dal profilo criminale $o$, nei casi più blandi, un comportamento trasgressivo di norme sociali.

Il disagio psicologico che è all'origine di tali strategie nascerebbe dunque dall'attrito con un sistema di valori dominanti.

La reazione di neutralizzazione non produce, né presuppone un sovvertimento dei valori comunemente accettati, ma anzi, in maniera solo apparentemente paradossale, implica di fatto un'accettazione del sistema di valori condivisi22. Vengono così superati i sensi di colpa e di vergogna ed il conflitto con la morale sociale, in modo da salvaguardare il sistema di valori comuni, altrimenti messi a rischio ${ }^{23}$.

La sensazione di riuscire a controllare la realtà tende a rinforzare il bisogno di persecuzione e, applicando le teorie di Pavolv ${ }^{24}$ e del rinforzo

Psychological Perspectives for Prevention, Policing and Treatment, John Wiley \& Sons, 2002.

${ }^{21}$ Matza D., Sykes G., "A Theory of Delinquency", in American Sociological Review, n. 22, 1957.

22 Ibidem.

${ }^{23}$ Ivi, pp. 646-670.

${ }^{24}$ Pavlov I. P., Conditioned Reflexes: An Investigation of the Physiological Activity of the Cerebral Cortex, Translated and Edited by G. V. Anrep., London, Oxford University Press, 1927. differenziale di Skynner ${ }^{25}$ e Lewin ${ }^{26}$, le probabilità di compiere un reato dipendono dal tipo di risposta allo stimolo.

"Il bisognoso d'affetto", invece, è quella tipologia di stalker motivata dalla ricerca di relazioni d'amore o, semplicemente, di semplici attenzioni d'affetto. Non a caso la vittima risulta essere, nella maggior parte dei casi, una persona vicina.

Questa del cercatore d'intimità risulta essere la forma di stalking più persistente ${ }^{27}$ e perpetrata maggiormente da donne con disturbi psicotici; in particolare, è prevalente il delirio di tipo erotomanico in cui il bisogno di affetto viene erotizzato e lo stalker tende a leggere nelle risposte della vittima un desiderio di resistenza. Il mero rifiuto o la paura dello stesso è vissuto come un'intollerabile attacco all'Io.

Questa categoria è quella che, almeno apparentemente, sembra investire il genere femminile autore di stalking e che, già nel lontano 1921, fece parlare $\mathrm{Hart}^{28}$ di "follia delle vecchie zitelle".

Secondo studi psichiatrici, ci sarebbero tre fasi rilevanti a questo proposito.

Una prima fase sarebbe quella della costruzione di un particolare spazio coscienziale strutturato dall'attesa dell'amore, che avrebbe lo scopo di riempiere il "gap" fra la percezione della propria limitatezza e l'aspirazione verso l'incontro, quale definitivo superamento di questa percezione. A

25 Skinner B.F., The behavior of organisms. New York: Appleton-Century-Crofts, 1938; ID, "A case history in scientific method", American Psychologist, 1956.

26 Lewin K., A dynamic theory of personality: Selected papers., New York, McGraw-Hill, 1935.

${ }^{27}$ Mullen P.E., Pathè M., Purcell R., Stuart G.W., “A study of stalkers", in American Journal of Psychiatry, 1999.

28 Hart B.,The Psychology of Insanity, Cambridge University Press, Cambridge, 1921. 
questo momento seguirebbe proprio quello dell'incontro, ovvero l'attesa, da cui deriverebbe la "spinta all'azione" volta alla conferma esterna del processo interno descritto.

In assenza di un segnale di disponibilità, si verifica il delirio erotomanico causato dal confronto tra la propria coscienza passionale e la realtà dell'altro 29 .

La patologia viene generata dalla convinzione di essere oggetto di attenzioni amorose di una persona, la quale, di riflesso, diviene l'unico soggetto d'amore e, quindi, come nella sindrome di Stoccolma, di ossessione ${ }^{30}$. La psiche crea un mondo parallelo e fantastico in cui gli elementi tipici di una relazione si manifestano, scatenando una reazione compulsiva nei confronti dell'oggetto desiderato in forza della quale la persona amata diviene vittima di persecuzioni ossessive $^{31}$.

Una terza tipologia di persecutore è quella definita "il corteggiatore incompetente", che tiene un comportamento alimentato dalla sua scarsa o inesistente competenza relazionale, che si traduce poi in comportamenti opprimenti, espliciti o anche aggressivi. Questa categoria sembrerebbe di minor efferatezza e sicuramente dotata di minore carica ossessiva. Rispetto agli altri tipi di stalking, le molestie del corteggiatore incompetente durano meno nel tempo poiché procurano allo stalker scarse soddisfazioni. Sembra però che questi sia il più recidivo di tutti. Spesso, infatti, i soggetti appartenenti a questa tipologia mettono in atto condotte di stalking nei confronti di più vittime e cercano un nuovo

29 Benvenuti P., Psicopatologia nell'arco della vita, Seid editori, Firenze, 2007; Sims A., Introduzione alla psicopatologia descrittiva, Raffaello Cortina, Milano, 2009. ${ }^{30} \mathrm{Ivi}$.

${ }^{31} \mathrm{Ivi}$. bersaglio quando non hanno avuto successo con quello precedente ${ }^{32}$.

Ancora diversa è la categoria del persecutore "respinto", il cui scopo è quello di ristabilire la relazione $\mathrm{o}$, in mancanza, di vendicarsi per l'abbandono, la cui persecuzione rappresenta una forma di relazione che rassicura rispetto alla perdita totale, percepita come intollerabile.

In questo "inseguitore assillante" gioca un ruolo cruciale il modello di attaccamento sviluppato ${ }^{33}$ in grado di scatenare angosce legate all'abbandono che creano una tendenza interiore, più o meno consapevole, a considerare l'assenza dell'altro come una minaccia di annientamento e di annullamento del sé.

Il mancato funzionamento dei modelli operativi interni non permetterà il corretto sviluppo ed il senso motorio di Piaget ${ }^{34}$, favorendo l'assimilazione, l'accomodamento $\mathrm{O}$ l'attaccamento patologico persecutorio.

L'adattamento individuale, così come lo vede Bowlby $^{35}$, è un processo continuo e attivo nel quale una persona reagisce e modella il proprio ambiente interpersonale. Bowlby, riprendendo direttamente Piaget ${ }^{36}$, sottolinea che l'esclusione difensiva riveste un ruolo determinante nelle relazioni di attaccamento in grado di trasformarsi in comportamenti non sani e quindi anche patologici.

\footnotetext{
32 Stefanelli M., "Lo stalker. Profili e trattamento", in Psicoterapeuti in formazione, n. 7, 2011.

${ }_{33}$ Bowlby J., Attaccamento e perdita, vol. 1: L'attaccamento alla madre, Boringhieri, Torino, 1972; ID, Attaccamento e perdita, vol. 2: La separazione dalla madre, Boringhieri, Torino, 1975; ID, Attaccamento e perdita, vol. 3: La perdita della madre, Boringhieri, Torino, 1983.

34 Piaget J., Lo sviluppo mentale del bambino e altri studi di psicologia, Einaudi, Torino 1967.

${ }^{35}$ Bowlby J., op. cit.

36 Piaget J., op. cit.
} 
Infine, è stata descritta una categoria di stalker definita "il predatore" e costituita da un molestatore che ambisce ad avere rapporti sessuali con una vittima che può essere pedinata, inseguita e spaventata. La paura, infatti, spinge e dirige questo tipo di stalker che prova un senso di potere nell'organizzare l'assalto ${ }^{37}$.

Questo genere di stalking è quello che colpisce anche i bambini perché posto in essere da persone con disturbi nella sfera sessuale.

A questo proposito Curcis8 utilizza l'espressione "sindrome delle molestie assillanti" proprio per descrivere le condotte indesiderate distinguendole in tre tipologie specifiche, delle comunicazioni indesiderate, dei contatti indesiderati e dei comportamenti associati, diversamente da Meloy e Gothard ${ }^{39}$, che utilizzano il termine "inseguitore ossessivo", in generale, per descrivere il soggetto che mette in atto comportamenti reiterati ed ostinati di persecuzione e molestia nei confronti di un'altra persona.

\section{La natura del reato e gli elementi costitutivi del delitto di stalking.}

Come Curci anche Mullen ${ }^{40}$ paragona lo stalking ad una costellazione di comportamenti riguardanti tentativi ripetuti e perduranti di ricercare contatto con un non consenziente.

\footnotetext{
${ }^{37}$ Mullen P.E., op. cit., 1999.

38 Curci P., Galeazzi G.M., Secchi C, La sindrome delle molestie assillanti, Torino, Bollati Boringhieri, 2003.

${ }^{39}$ Meloy J.R., Gothard S., "A demografic and clinical comparison of obsessional followers and offenders with mental disorders", American Journal of Psychiatry, 1995.

${ }^{40}$ Mullen P.E., Pathè M., Purcell R., Stuart G.W., op. cit, 1999.
}

In effetti il perdurante e grave stato di ansia o di paura ${ }^{41}$, unitamente al fondato timore per l'incolumità propria, di un prossimo congiunto ovvero di un altro soggetto legato da una relazione affettiva e la necessità del bisogno di mutare le abitudini di vita ${ }^{42}$, costituiscono gli elementi del reato di stalking, che distinguono e qualificano quest'ultimo rispetto alle minacce ex art. $612 \mathrm{cp}$.

Inserito tra i "delitti contro la libertà morale", come scritto da Fiandaca ${ }^{43}$, lo stalking vuole tutelare la libertà morale e la libertà di autodeterminarsi in tutti gli aspetti della vita personale e quotidiana, salvaguardando ${ }^{44}$ la libertà di movimento, la libertà economica e a tutte le altre libertà, inevitabilmente pregiudicate $^{45}$. Non è un caso che il concetto di autodeterminazione si inserisca all'interno del concetto più ampio di libertà psichica, di inviolabilità e di tranquillità collettiva.

\footnotetext{
${ }^{41}$ A tal proposito la Corte di Cassazione $\mathrm{V}^{\circ}$ sez. pen. n. 20531 del 2014 ha ribadito che non è necessario che il turbamento emotivo della vittima determini in essa un vero e proprio stato patologico, essendo sufficiente che gli atti persecutori abbiano determinato, in quest'ultima, l'insorgere di un'alterazione dell'equilibrio psicologico.

42 Quali ad esempio l'essere costretti a cambiare proprio numero di cellulare, o il non frequentare più gli abituali luoghi di ritrovo e svago o il modificare la strada usuale e più in generale le proprie consuetudini personali e di lavoro.

${ }^{43}$ Fiandaca G. e Musco E., Diritto penale. Parte speciale, Vol. II, Zanichelli, Bologna, 2014, tomo primo.

${ }^{44}$ ID, I delitti contro la persona, Zanichelli, Bologna, 2011.

45 Tutte cioè le libertà inevitabilmente pregiudicate dall'aggressione alla libertà morale e le libertà bisognose di protezione, dalla libertà di movimento alla libertà economica. Libertà intesa dunque come un bene valore assoluto ed anticipato, in senso figurato, rispetto a tutte le altre forme di libertà. Senza libertà morale appare difficile concepire una libertà di pensiero, di coscienza, di affetto, di vita di relazione, di vita sessuale, di determinazione, Ivi, p.200.
} 
Oltre Fiandaca e Musco ${ }^{46}$, anche Marino ${ }^{47}$, Maugeri $^{48}$ e Lo Monte ${ }^{49}$ relativamente alla tranquillità individuale ${ }^{50}$ sostengono che l'insorgere dello stato di paura e di ansia, ovvero il fondato timore per l'incolumità, costituiscono sicuramente una lesione alla serenità psicologica, alla tranquillità personale.

Si perfeziona così un sistema di protezione a tutela progressiva che, a dire di Bricchetti e Pistorelli51, ha contribuito a generare un reato plurioffensivo con tutela anticipata, rispetto alla possibile commissione di reati più gravi.

La protezione teleologica ${ }^{52}$, come confermato da Coco e da Peccioli53, mira a verificare il collegamento tra le condotte persecutorie realizzate dallo stalker ed il verificarsi di uno dei tre presupposti richiesti dall'art. 612 bis $\mathrm{cp}^{54}$.

La particolarità del reato de quo è che le conseguenze psicologiche riportate dalla vittima devono risultare intrinsecamente legate allo stato di ansia e al fondato timore per la propria o

\footnotetext{
${ }^{46} \mathrm{ID}$, op. cit.

47 Marino R., Violenza sessuale, pedofilia, stalking, Simone, Napoli, 2009.

48 Maugeri A.M., Lo stalking tra necessità politico-criminale e promozione mediatica, Giappichelli, Torino, 2010.

49 Lo Monte E., "Art. 612 bis cp e Stalking occupazionale: un rimedio solo apparente", in Lavoro e previdenza oggi, 2009, I, 1; ID, "Una nuova figura criminosa: lo stalking", in Indice penale, 2010, II, p. 479.

${ }^{50} \mathrm{Da}$ ultimo la sentenza Corte di Cassazione $\mathrm{III}^{\circ}$ sez. pen, n. 25889 del 2013.

51 Bricchetti R., Pistorelli L., "Violenza sessuale e stalking: commento al D.L. 23 febbraio 2009 n. 11 convertito con modifiche in L. 23 aprile 2009 n 38", in Guida diritto, fasc.10, 2009 , p. 7.

52 Il reato de quo è un reato comune, abituale, di evento, ma soprattutto di danno.

${ }^{53}$ Coco P., La tutela della libertà individuale nel nuovo sistema anti- stalking, Jovine, Napoli, 2012; Cesari F., "Custodia in carcere per il marito molestatore. Prime applicazioni del reato di stalking", in Fam. e dir., 2009; Peccioli A., "Stalking: bilancio di un anno dall'entrata in vigore", in Dir. pen. e proc., 2010.
}

altrui incolumità, costituendo indici individuali interni, e come tali di difficile risvolto probatorio perché accertabili prevalentemente mediante parametri clinico-forensi ${ }^{55}$.

Più semplice risulta invece la prova della modificazione delle abitudini personali o professionali causate dal comportamento reiterato dello stalker.

Nel mondo del diritto si dice che il nesso della causalità psichica genera delle serie impossibilità a predeterminare le azioni umane alla luce di stimoli provenienti da terzi e rende impossibile il ricorso ad un criterio di probabilità statistica per legare causalmente condotta ed evento.

La ricerca del legame eziologico avviene generalmente ${ }^{56}$ con una totale equiparazione tra cause fisiche e cause psicologiche dell'evento, facendo riferimento ad influssi che condizionano significativamente lo spazio di autodeterminazione della vittima, riducendolo e in taluni casi annullandolo del tutto ${ }^{57}$.

A questo proposito Cornacchia ${ }^{58}$ chiarisce che, rifacendosi al concetto di causa efficiente di Aristotele, basterebbe dimostrare l'evento

${ }^{54}$ Corte di Cassazione V ${ }^{\circ}$ sez. pen. no 20993 del 2012 e Corte di Cassazione III $^{\circ}$ sez. pen. $n^{\circ} 15334$ del 2013.

55 In tal senso vi è da ultimo una sentenza della Corte di Cassazione $\mathrm{V}^{\circ}$ sez. pen. n. 16864 del 2011 secondo cui non è assolutamente previsto un accertamento di uno stato patologico della vittima, essendo sufficiente verificare che la condotta persecutoria dello stalker abbia prodotto un effetto destabilizzante per la serenità e l'equilibrio psicologico della stessa, proprio perché la fattispecie dello stalking è una previsione normativa diversa dal reato di lesioni personali.

${ }^{56}$ Caputo M., "Eventi e sentimenti nel delitto di atti persecutori", in Bertolino M., Eusebi L., Forti G, (a cura di), Studi in onore di Mario Romano, vol. III, Jovene, Napoli, 2011.

${ }^{57} \mathrm{Ivi}$, p. 1400.

58 Cornacchia L., "Il problema della c.d. causalità psichica rispetto ai condizionamenti mentali", in AAVV, Nuove esigenze di tutela nell'ambito dei reati contro la persona, Zanichelli, Bologna, 2001, p. 205 e ss. 
"antecedente" dal punto di vista cronologico, mentre Risicato59 richiama il classico "id quod plerumque accidit'.

Questa interpretazione sembra essere più conforme alla natura abituale degli atti persecutori, poiché questi ultimi normalmente concentrano il proprio disvalore sulla condotta della quale si richiede la reiterazione, potendosi adattare anche al reato di danno in cui ciò che assume rilievo non è la reiterazione delle condotte offensive, bensì la verificazione dell'evento con cui si realizza la lesione del bene giuridico ${ }^{60}$.

Maugeri $^{61}$ sottolinea che così ragionando si rispetterebbe anche in toto il principio di offensività, visto che si valorizza l'accertamento dell'humus della condotta, richiedendo un'oggettiva carica offensiva in termini di idoneità 62 .

\section{Il rapporto persecutorio, la relazione} disturbata e i danni sulla vittima di stalking. I danni subiti dalla vittima sembrano diversificarsi a seconda delle singole reazioni ai comportamenti persecutori ed, in concreto, variano in ragione delle differenti caratteristiche della vittima.

\footnotetext{
59 Risicato L., La causalità psichica tra determinazione $e$ partecipazione, Giappichelli, Torino, 2007, p. 73 ss.

${ }^{60}$ Così Maugeri A.M., op.cit., p. 155.

${ }^{61} \mathrm{Ivi}, \mathrm{p} .156$.

${ }^{62}$ Anche la Cassazione ha statuito che il delitto di atti persecutori è reato ad evento di danno e si distingue sotto tale profilo dal reato di minacce, che è reato di pericolo, in Corte di Cassazione, $\mathrm{V}^{\circ}$ sez. pen., $\mathrm{n}$. 17698 del 2010, ed anche Corte di Cassazione, $V^{\circ}$ sez. pen. n. 14391 del 2012 e Corte di Cassazione $V^{\circ}$ sez. pen. n. 20993 del 2013, Tribunale di Bari, sez. riesame, 6 aprile 2009 (ord.), in Giur. Merito, 2009, p. 1921 ss.
}

Alcuni studi sul fenomeno ${ }^{63}$ hanno mostrato dei risultati interessanti in tema, soprattutto considerando la relazione patologica che si instaura con la vittima.

Esisterebbe, ad esempio, una categoria sociale a rischio rappresentata da tutti gli appartenenti alle cosiddette professioni d'aiuto (medici, psicologi, infermieri) con cui il contatto tra stalker e vittima diventa una richiesta di affetto o di attenzione o, in alcuni casi, anche di vendetta. Esisterebbero momenti di lucidità dello stalker a cui si sostituiscono momenti di ansia e stress che inducono ad agire da stalker. Se alla consapevolezza di essere stati lasciati, o disprezzati, o non curati, segue un comportamento che vuole nutrirsi del torto subito, allora si parla già di agire ossessivo ${ }^{64}$.

Il delirio riparatorio e la conseguente cronicizzazione del sentimento manifestano dei comportamenti ossessivi e di disturbo della personalità che spingono lo stalker ad agire compulsivamente, negando la realtà, danneggiando progressivamente la propria salute mentale e la qualità della propria vita sociale, che si deteriora, arrivando alla persecuzione vera e propria.

Inutile sottolineare che la vittima di stalking subisce un danno psichico $^{65}$ la cui

63 Meloy J. R., The psychology of stalking, Academic Press, 1998; ed anche Mullen P.E., Pathè M., Purcell R., Stuart G., op. cit, 1999; Oliviero Ferraris A., "Stalker il persecutore", in Psicologia Contemporanea, 2001.

${ }^{64}$ Fabbroni B, Giusti A., Vittima persecutore. Il mondo dello stalker, Edizioni universitarie romane, Roma, 2009.

${ }^{65}$ Secondo i criteri del DSM IV-TR, la caratteristica principale del Disturbo Post Traumatico da Stress (DPTS) è da ricercarsi nello sviluppo di sintomi tipici che seguono l'esposizione ad un fattore traumatico, che causa o può comportare a volte anche la morte o le lesioni personali. Tale disturbo comporta inoltre da parte della vittima di stalking un ripercorrere l'evento 
ristrutturazione diviene di competenza specialistica $^{66}$ per l'analisi delle probabili reazioni dello stalker e dei prevedibili danni fisici, psicologici e di relazione.

Nei trattamenti Evidence-Based ${ }^{67}$ dovuti al disturbo post-traumatico acuto da stress, le vittime di stalking sono aiutate grazie all'elaborazione emotiva degli episodi subiti, ove la reale minaccia si sia ridimensionata ${ }^{68}$. La tecnica EMDR, ad esempio, in questo riesce a dare un valido aiuto e una risoluzione elevata, agendo direttamente sull'esperienza vissuta, eliminandone i componenti emotivi disturbanti ${ }^{69}$.

traumatico attraverso sogni spiacevoli, ricordi ricorrenti ed intrusivi come se il fatto si stesse ripresentando, determinando quindi nel soggetto un intenso e logorante disagio psicologico., Andreoli V., DSM IV TR, Criteri diagnostici, American Psychiatric Association, Masson, England, 2000.

66 Generalmente quando si sceglie di risolvere il conflitto ricorrendo a tecniche di counseling vittimologico le linee guida principali chiedono alla vittima di interrompere qualunque contatto con lo stalker, a mezzo telefono, posta, posta elettronica, o semplicemente contatti de visu, perché qualsiasi reazione può comportare anche un aggravamento e rafforzare il comportamento dello stalker.

${ }^{67}$ EBT, Trattamenti basati su prove di efficacia, in cui la pratica basata sulle evidenze è data dall'integrazione della ricerca con la pratica clinica, nel rispetto delle caratteristiche del paziente, della sua cultura e delle sue opinioni. L'approccio Evidence-Based Practice si contrappone alle regole basate sul "buon senso", identificando le prove a sostegno di uno specifico trattamento e valutando la loro validità scientifica. Il suo obiettivo è eliminare trattamenti malsani o eccessivamente rischiosi a favore di quelli che danno risultati migliori. In tale sede vengono anche definiti $\mathrm{i}$ tre livelli di evidenza scientifica.

${ }^{68} \mathrm{Il}$ sostegno multilivello, oltre a fornire un appoggio alla persona o un semplice luogo di ascolto, che riduca il senso di isolamento e solitudine, rinforzando le difese e, riducendo lo stato di terrore, si realizza mediante un percorso multifunzionale che, dopo la presa di coscienza, intenda studiare e realizzare le migliori strategie funzionali alla vita della vittima, sia per la sua ri-costruzione individuale che per quella sociale.

${ }^{69}$ Inizialmente usato per il trattamento del Disturbo Post Traumatico da Stress, attualmente, questo metodo terapeutico apre una nuova dimensione nella
Maugeri specifica che il diverso tipo di comportamento dello stalker e la sua frequenza, di fatto, determinano reazioni esclusivamente soggettive, totalmente dipendenti dalla personalità della vittima, dalle sue esperienze e soprattutto dal contesto culturale di riferimento ${ }^{70}$.

Se in un primo momento gli stalkers sembravano essere esclusivamente di genere maschile, oggi si registra la presenza di numerose donne atte a tenere comportamenti ossessivi nei riguardi di uomini, come registrato dall'Osservatorio nazionale stalking ${ }^{71}$.

Secondo gli indici Istat, nel 2009 le querele per stalking sono state 2.023 e di queste 873 sarebbero state archiviate per mancanza di prove, insussistenza del fatto o perché l'autore è riuscito a rimanere ignoto.

Dal 2009, secondo i dati del Centro presunti autori, sembra che ci sia stato un aumento del $30 \%$ di denunzie maschili, rispetto a quelle precedenti l'entrata in vigore del delitto degli atti persecutori $^{72}$.

Come definito dalla Convenzione del Consiglio $\mathrm{d}^{\prime}$ Europa $^{73}$ su "prevenzione e lotta contro la violenza nei confronti delle donne e la violenza domestica" siglata a Istanbul nel 2011, lo stalking

psicoterapia per l'efficacia dimostrata in tutti i tipi di trauma. Le ricerche condotte su vittime di violenze sessuali, di incidenti, di catastrofi naturali, indicano che il metodo permette una desensibilizzazione rapida nei confronti dei ricordi traumatici e una ristrutturazione cognitiva che porta a una riduzione significativa dei sintomi del paziente (stress emotivo, pensieri invadenti, ansia, flashbacks, incubi).

${ }^{70}$ Maugeri A.M., op.cit., p. 155.

71 Disponibile alla pagina del sito dell'Osservatorio nazionale stalking e centro presunti autori di Massimo Lattanzi: www.stalking.it.

$\begin{array}{lccc}72 & \text { Disponibile } & \text { alla } & \text { pagina: } \\ \text { www.centropresuntiautori.it. } & & \\ 73 & \text { Disponibile } & \text { alla } & \text { pagina: }\end{array}$
www.coe.int/conventionviolence. 
femminile è sottile, subdolo, fatto di minacce velate e di numerose violenze psicologiche. A differenza dello stalking maschile, predilige l'accanimento e la persecuzione su un individuo specificamente analizzato. La stalker metterebbe in atto le tecniche più sofisticate per elaborare strategie di attacco, ma anche difensive per sviare indagini da se stessa e per mettere la vittima in totale difficoltà probatorio.

Sembra che le donne compiano delle azioni di minore gravità, ma di maggior astuzia ${ }^{74}$, richiamando alla mente la donna mostro di Cesare Lombroso ${ }^{75}$. La violenza fisica non costituirebbe dunque un fattore caratterizzante la gravità dell'azione, ma avrebbe un impatto psicologico decisamente invalidante per l'uomo vittima $^{76}$.

Nei diversi periodi storici emerge un quadro patologico della stalker piuttosto stabile.

Secondo Purcell, Pathé e Mullen ${ }^{77}$ le stalkers appaiono molto simili agli uomini, con un'unica differenza nella scelta della vittima. Mentre le donne indirizzano i loro comportamenti persecutori verso individui determinati e per specifici motivi, gli uomini, solitamente, si orienterebbero verso vittime del sesso opposto per ragioni più comuni.

\footnotetext{
${ }^{74}$ Sinclair H. C., Frieze I. H., "Initial courtship behaviour and stalking: How should we draw the line?", in Davis J. A., Frieze I.H., Maiuro R.D., Stalking: Perspectives on victims and perpetrators, Springer Publishing Company, New York, 2002.

${ }^{75}$ Lombroso C., Ferrero G., La donna delinquente, la prostituta, e la donna normale, Fratelli Bocca, Milano, 1903.

${ }^{76}$ Thomas S. D. M., Purcell R., Pathè M., Mullen, P. E., "Harm associated with stalking victimization", Australian and New Zealand Journal of Psychiatry, 2008.

${ }^{77}$ Mullen P.E., Pathè M., Purcell R., Stuart G.W., op. cit, 1999.
}

Meloy e Boyd ${ }^{78}$ hanno fatto emergere il profilo preciso di una donna stalker, quale donna single, eterosessuale, istruita, intorno ai 30 anni, separata o divorziata, caratterizzata dall'avere un disturbo di personalità borderline, che è stato poi spiegato da Meloy, da Mohandie e McGowan ${ }^{79}$ con una propria diagnosi psichiatrica.

Diventano stalker le donne con disturbi dell'umore la cui necessità di persecuzione si manifesterebbe soprattutto nelle relazioni sentimentali in cui vincerebbero le ossessioni dell'angoscia dell'abbandono, della smania di possesso o di rifiuto.

Lo stalking è principalmente un comportamento intrusivo, controllante e reiterato nel tempo, rivolto ad una o più persone ritenute desiderabili ed inavvicinabili ed il disturbo borderline ne rappresenta il versante psicotico, limitando la libertà ed al contempo soddisfacendo il desiderio di vicinanza e possesso ${ }^{80}$.

Il comunissimo disturbo borderline che caratterizzerebbe l'uomo stalker è presente anche nella personalità della figura femminile, sicuramente causato da un vissuto emozionale eccessivo e variabile.

Infatti, anche secondo la teoria biopsicologica di M. Linehan ${ }^{81}$, il cuore del disturbo borderline di personalità, che in generale corrisponde alla disregolazione emotiva causata dall'interazione tra la vulnerabilità emotiva da una parte e da un ambiente invalidante dall'altra, potrebbe anche

78 Meloy J., Boyd C., "Female stalkers and their victims", Journal of the American Academy of Law and Psychiatry, 2003.

${ }^{79}$ Mohandie K., Meloy JR., McGowan M., Williams J., "The recon typology of stalking: reliability and validity based upon a large sample of North American stalkers", J Forensic Sci, 2006.

${ }^{80} \mathrm{Ivi}$.

${ }^{81}$ Lineahn M., Trattamento cognitivo comportamentale del disturbo borderline, Raffaello Cortina, Milano, 2011. 
essere causato da un rapporto eccessivamente ossessivo con l'altro.

Secondo Bushman ed altri, la ruminazione rabbiosa è uno stile maladattivo di pensiero che si attiva in presenza di emozioni di rabbia, focalizzando l'attenzione su quest'ultima, sulle sue cause e sulle sue conseguenze, alimentando l'attivazione emotiva negativa e aumentando la tendenza a rispondere con comportamenti aggressivi o paranoidi ${ }^{82}$.

Lo stesso attaccamento, se viziato ed eccessivo, come spiegato da Bowlby, potrebbe ingenerare la nascita di fenomeni persecutori.

Non tutti gli stalkers sono dotati della stessa carica offensiva e di fatto ledono seriamente la loro vittima.

Cupach e Spitzberg ${ }^{83}$ distinguono lo stalker molestante, che cerca affetto ed intimità con la vittima, dallo stalker intrusivo, che invece cerca l'intimità con tattiche strumentali, manipolative ed opportunistiche.

A questo proposito i due studiosi spiegano la differenza tra lo stalker organizzato, che è alla ricerca di una vendetta personale e perciò causa danni, pianificando con cura le modalità di persecuzione, dallo stalker disorganizzato, che al contrario è un soggetto arrabbiato e vendicativo, sicuramente non in condizione di pianificare in modo strategico la sua persecuzione.

Secondo Rosenfeld ${ }^{84}$, relativamente al rapporto amore - vendetta e al disturbo psicologico del persecutore, si possono distinguere quattro categorie di stalker.

\footnotetext{
${ }^{82}$ Bushman B., Roy Baumeister F., Social Psycology and human nature, Comprehensive edition, 2010.

${ }^{83}$ Cupach W. R.,Spitzberg B.H., Attrazione, ossessione e stalking, Astrolabio Ubaldini, Roma, 2011.
}

Lo stalker borderline e con disturbo psicotico dell'umore, che ha con la vittima una vera relazione reale, diversa dello stalker paranoide ed antisociale, che vive una relazione di pura fantasia, priva di danno.

In quest'ultima tipologia si evidenziano gli stalkers con disturbo delirante e con disturbo psicotico-erotomane dell'umore, e gli stalkers affetti da disturbo delirante con disturbo psicotico persecutorio.

Come spiegato ulteriormente da Boon e Sheridan ${ }^{85}$ esiste una relazione persecutoria che gradua il rischio di reato in base al rapporto che si ha con la vittima, a seconda cioè che vi sia stata o meno una relazione, e se questa sia di maggiore o minore durata.

Si passerebbe dunque dal controllo possessivo sulla vita della vittima ad uno più innocuo, distinguendo, quindi, gli stalkers di alto rischio da quelli di basso o innocuo rischio ${ }^{86}$.

A seconda del comportamento realizzato e soprattutto del danno subito, la vittima potrà essere qualificata come tale. Deve dunque essere provata la sofferenza e la graduazione della stessa, indicativa della responsabilità penale, che genera effetti e conseguenze sullo stile di vita e sul suo benessere invalidato non solo nella sfera affettiva, ma anche nella salute cognitiva, fisica, sociale, spirituale e talvolta economica.

Il disturbo arrecato, che possa trasformarsi in trauma $\mathrm{o}$ in semplice nocumento $\mathrm{o}$ stress, sarà quantificato oltre che in sede penale, anche in sede civile, quale forma di risarcimento danni.

\footnotetext{
${ }^{84}$ Rosenfeld B., "Violence risk factors in stalking and obsessional harassment", in Criminal Justitia and Behavior, vol. 31, 2004.

${ }^{85}$ Fabbroni B. e Giusti M.A., op. cit.

${ }^{86} \mathrm{Ivi}$.
} 
Di recente una sentenza del Tribunale di Genova $^{87}$ ha riconosciuto gli estremi del reato di cui all'art 612 bis cp, applicando il regime di tolleranza zero ad atti persecutori avvenuti all'interno di un condominio ${ }^{88}$.

Già la Cassazione penale 89 aveva esteso nel 2011 l'ambito di applicabilità dell'art. 612 bis cp al contesto condominiale, applicando un'interpretazione lata della norma, sancendo che l'applicazione dello stalking potrebbe essere un efficace strumento di tutela anche per tutti coloro che in via indiretta subiscono un turbamento alla propria tranquillità domestica e sono o si sentono costretti ad alterare il proprio modus vivendi ${ }^{90}$.

\section{Bibliografia.}

- Alfarano E., La Tegola D., Carabellese F., Catanesi R., "Stalking al femminile: una review di letteratura", Rassegna italiana di Criminologia, anno IV, n.1, 2012.

- Andreoli V., DSM IV TR, Criteri diagnostici, American Psychiatric Association, Masson, England, 2000.

- Benvenuti P., Psicopatologia nell'arco della vita, Seid editori, Firenze, 2007.

- Bowlby J., Attaccamento e perdita, vol. 1: L'attaccamento alla madre, Boringhieri, Torino, 1972.

- Bowlby J., Attaccamento e perdita, vol. 2: La separazione dalla madre, Boringhieri, Torino, 1975.

\footnotetext{
87 Tribunale di Genova n. 20895 del 2011.

88 Lo stalking condominiale, che non è un'ipotesi speciale codificata dal Legislatore, bensì il frutto di una particolare applicazione giurisprudenziale della figura criminosa, è reso possibile dalla non del tutto tassativa formulazione degli elementi costitutivi della fattispecie e sta trovando terreno fertile in una realtà da sempre foriera di contrasti e dissidi che può dirompere nell'area del penalmente rilevante, qualora vengano lesi o messi in pericolo beni giuridici tutelati da specifiche fattispecie incriminatrici.

${ }^{89}$ Corte di Cassazione $V^{\circ}$ sez. pen. , n. 20895 del 2011.

${ }^{90} \mathrm{Ivi}$.
}

- Bowlby J., Attaccamento e perdita, vol. 3: La perdita della madre, Boringhieri, Torino, 1983.

- Bricchetti R., Pistorelli L., "Violenza sessuale e stalking: commento al D.L. 23 febbraio 2009 n. 11 convertito con modifiche in L. 23 aprile 2009 n 38", Guida diritto, fasc. 10, 2009.

- Bushman B., Roy Baumeister F., Social Psycology and buman nature, Comprehensive edition, 2010.

- Caputo M., "Eventi e sentimenti nel delitto di atti persecutori", in Bertolino M., Eusebi L. e Forti G. (a cura di), Studi in onore di Mario Romano, vol. III, Jovene, Napoli, 2011.

- Cesari F., "Custodia in carcere per il marito molestatore. Prime applicazioni del reato di stalking", Fam. e dir., 2009.

- Coco P., La tutela della libertà individuale nel nuovo sistema anti-stalking, Jovine, Napoli, 2012.

- Cornacchia L., "Il problema della c.d. causalità psichica rispetto ai condizionamenti mentali", in AAVV, Nuove esigenze di tutela nell'ambito dei reati contro la persona, Zanichelli, Bologna, 2001.

- Cupach W. R. e Spitzberg B.H., Attrazione, ossessione e stalking, Astrolabio Ubaldini, Roma, 2011.

- Curci P., Galeazzi G.M., Secchi C, La sindrome delle molestie assillanti, Torino, Bollati Boringhieri, 2003.

- Dressing, H., Kuehner, C., \& Gass P., "Prevalence of stalking in Germany", Psychiatrische Praxis, 32 (2), 2005 a.

- Dressing, H., Kuehner, C. \& Gass, P., "Lifetime prevalence and impact of stalking in a European population: epidemiological data from a middle-sized German city", British Journal of Psychiatry, 187, 2005 b.

- Dressing H., Kuehner C., Gass, P., "The epidemiology and characteristics of stalking", Current opinion in psychiatry, 19 (4), 2006.

- Fabbroni B., Giusti M.A., Vittima persecutore. Il mondo dello stalker, Edizioni universitarie romane, Roma, 2009.

- Fiandaca G., Musco E. , Diritto penale. Parte speciale, Vol. II, Zanichelli, Bologna, 2014.

- Fiandaca G., Musco E. , I delitti contro la persona, Zanichelli, Bologna, 2011.

- Hart B., The Psychology of Insanity, Cambridge University Press, Cambridge, 1921. 
- Lewin, K., A dynamic theory of personality: Selected papers, New York: McGraw-Hill, 1935.

- Lineahn M., Trattamento cognitivo comportamentale del disturbo borderline, Raffaello Cortina, Milano, 2011.

- Lo Monte E., "Art. 612 bis cp e stalking occupazionale: un rimedio solo apparente", Lavoro e previdenza oggi, I, 1, 2009.

- Lo Monte E., "Una nuova figura criminosa: lo stalking", Indice penale, II, 2010.

- Lombroso C., Ferrero G., La donna delinquente, la prostituta, e la donna normale, Fratelli Bocca, Milano, 1903.

- Marino R., Violenza sessuale, pedofilia, staking, Simone, Napoli, 2009.

- Matza D., Sykes G., "A Theory of Delinquency", American Sociological Review, n. 22, 1957.

- Maugeri A.M., Lo stalking tra necessità politicocriminale e promozione mediatica, Giappichelli, Torino, 2010.

- Meloy J., Boyd C., "Female stalkers and their victims", Journal of the American Academy of Law and Psychiatry, 2003.

- Meloy J. R., The psychology of stalking, Academic Press, 1998.

- Meloy J.R., Gothard S., "A demografic and clinical comparison of obsessional followers and offenders with mental disorders", American Journal of Psychiatry, 1995.

- Mohandie K, Meloy JR, McGowan M., Williams J., "The recon typology of stalking: reliability and validity based upon a large sample of North American stalkers", J Forensic Sci, 2006.

- Mullen P.E., "The victim of stalking", in Boon J, Sheridan L (Eds.), Stalking and Psychosexual Obsession: Psychological Perspectives for Prevention, Policing and Treatment, John Wiley \& Sons, 2002.

- Mullen P.E., Pathè M., Purcell R., Stalkers and their victims, Cambridge University 2000.

- Mullen P.E., Pathè M., Purcell R., Stuart G.W., "A study of stalkers", American Journal of Psychiatry, 1999.

- Oliviero Ferraris A., "Stalker il persecutore", Psicologia Contemporanea, 2001.

- Pavlov, I. P., Conditioned Reflexes: An Investigation of the Physiological Activity of the Cerebral Cortex. Translated and Edited by G. V. Anrep., Oxford University Press, 1927.

- Peccioli A., "Stalking: bilancio di un anno dall'entrata in vigore", Dir. pen. e proc., 2010.
- Piaget J., Lo sviluppo mentale del bambino e altri studi di psicologia, Einaudi, Torino 1967.

- Risicato L., La causalità psichica tra determinazione e partecipazione, Giappichelli, Torino, 2007.

- Rosenfeld B., "Violence risk factors in stalking and obsessional harassment", Criminal Justitia and Behavior, vol. 31, 2004.

- Sheridan L., Davies G., \& Bonn J., "The Course and Nature of Stalking: A victim Perspective", The Howard Journal, 2001.

- Sims A., Introduzione alla psicopatologia descrittiva, Raffaello Cortina, Milano, 2009.

- Sinclair H. C., Frieze I. H., "Initial courtship behaviour and stalking: How should we draw the line?", in Davis J. A., Frieze I.H., Maiuro R.D., Stalking: Perspectives on victims and perpetrators, Springer Publishing Company, New York, 2002.

- Skinner B.F., "A case history in scientific method", American Psychologist, 1956.

- Skinner B.F., The behavior of organisms, Appleton-Century-Crofts, New York, 1938.

- Spitzberg B., "The state of the art of stalking: taking stock of the emerging literature", Aggression and Violent Behavior, 2007.

- Stefanelli M., "Lo stalker. Profili e trattamento", Psicoterapeuti in formazione, n.7, 2011.

- Thomas S. D. M., Purcell, R., Pathè, M., Mullen, P. E., "Harm associated with stalking victimization", Australian and New Zealand Journal of Psychiatry, 2008.

\section{Giurisprudenza.}

- Tribunale di Bari, sez. riesame, 6 aprile 2009, in Giur. Merito, 2009.

- Tribunale di Genova n. 20895 del 2011.

- Corte di Cassazione, IV ${ }^{\circ}$ sez. pen. n. 2967 del 1978.

- Corte di Cassazione, $\mathrm{V}^{\circ}$ sez. pen., n. 17698 del 2010.

- Corte di Cassazione $\mathrm{V}^{\circ}$ sez. pen. n. 16864 del 2011.

- Corte di Cassazione Vºez. pen. , n. 20895 del 2011.

- Corte di Cassazione, $\mathrm{V}^{\circ}$ sez. pen. n. 14391 del 2012.

- Corte di Cassazione Vº sez. pen. $\mathrm{n}^{\circ} 20993$ del 2012.

- Corte di Cassazione $\mathrm{III}^{\circ}$ sez. pen, n. 25889 del 2013. 
- Corte di Cassazione $V^{\circ}$ sez. pen. n. 20993 del 2013.

- Corte di Cassazione III $^{\circ}$ sez. pen. nº 15334 del 2013.

- Corte di Cassazione $\mathrm{V}^{\circ}$ sez. pen. n. 20531 del 2014.

\section{Sitografia.}

- www.centropresuntiautori.it

- www.coe.int/conventionviolence

- www.istat.it

- www.istat.it/giustizia/sicurezza

- www.stalking.it 